\title{
An analysis of tactile letter confusions
}

\author{
TADASHI KIKUCHI, YUKIO YAMASHITA, KEN SAGAWA, and TENJI WAKE \\ Industrial Products Research Institute, Ibaragi 305, Japan
}

\begin{abstract}
A confusion matrix of the whole block capital letters of the alphabet was obtained so as to examine the nature of tactile letter recognition, using a 17 by 17 matrix of tactile stimulators placed against blind subjects' backs. A hierarchical cluster analysis and a nonmetric multidimensional scaling technique were applied to the matrix. The results of the two analyses were consistent with each other and indicated that at least three independent basic letter features-enclosing shapes, vertical parallel lines, and angle of lines-play important parts in tactile letter recognition. Most confusion may be attributable to displacement of the apparent loci, omission or fusion of loci of stimulation, and failure to detect gaps in the tactile letters.
\end{abstract}

Attempts to develop ways to recognize visual information through transformation onto the skin have been made by many investigators. Geldard (1966) developed a tactile coding system called the Optohapt, which converts printed or typed characters into tactile signals by means of nine vibrators scattered over the entire body as widely as possible. Bliss and his associates (Linvill \& Bliss, 1966) developed a reading-aid device, called the Optacon, which converts printed materials into vibratory patterns. A packed 24 by 6 tactile array is presented to one finger. Bach-y-Rita (1972) and Collins (1970) developed the Tactile Vision Substitution System (TVSS), in which a visual image is transformed into a tactile one by means of a 20 by 20 matrix of vibrators mounted on the back of a dental chair. These latter devices directly transform optical patterns into tactile ones, so that the topology of optical images is preserved.

Loomis (1974) studied tactile letter recognition with different modes of stimulus presentation. Block capital letters were displayed on the back of the subject, using the 400-point TVSS. He reported the confusion matrices and found that the best recognition accuracy was obtained when the slit modes of presentation were employed. ${ }^{1} \mathrm{He}$ concluded that the identification performance was the result of the limited spatial resolution of the skin. With a 10 by 10 array of vibrators called the Kinotact, Craig (1974) carried out tactile letter recognition and suggested that the decisions of subjects were based on some particular features of the letters, especially the edges.

The present study is concerned with the problem of tactile letter recognition. One purpose was to find the kinds of information blind people use in recognizing tactile letters presented to their backs. Another

Projects from the Agency of Industrial Science and Technology. The authors' mailing address: Industrial Products Research Institute, 1-1-4, Higashi, Yatabe-Machi, Tsukuba-Gun, Ibaragi-Ken 305, Japan. was to investigate the nature of the mutual interaction of multiple point stimulators when blind subjects try to recognize tactile letters. A confusion matrix was obtained as a device for answering some of the questions.

\section{METHOD}

\begin{abstract}
Apparatus
Briefly, the visual display captured by a stationary camera was converted into a 32 by 32 optical matrix. The central 17 by 17 matrix activated the corresponding tactors of a 17 by 17 matrix of tactile stimulators mounted on the back of a wheelchair. The dark region of the visual display activated the tactors in the corresponding areas of the tactile matrix, while the bright region of the visual display did not. The size of each tactor was $1.8 \mathrm{~mm}$ in diameter, and the vertical as well as the horizontal spacing (center to center) of the tactors was $16 \mathrm{~mm}$. The air-driven tactors vibrated at $2.5 \mathrm{~Hz}$, with a rise time of $50 \mathrm{msec}$, a fall time of $40 \mathrm{msec}$, and a pulse duration of $200 \mathrm{msec}$. The displacement depth of the tactors was $3.8 \mathrm{~mm}$. This particular vibration frequency was used in order to ensure strong stimulation. Thus it might be said that tactile, rather than vibrotactile, pressure stimulation was employed in this experiment.
\end{abstract}

\section{Stimuli}

Black block capital letters of the alphabet, drawn individually on white cardboard squares, were used as the visual display. The width of the lines of the letters was $20 \mathrm{~mm}$. These letters were converted into tactile letters. Each tactor in the tactile matrix corresponded to an area of $10 \times 10 \mathrm{~mm}$ on the cardboard. Thus a black line of the visual display activated a line two tactors wide in the tactile matrix. The letters used are shown in Figure 1 in dot format.

\section{Subjects}

Four blind subjects served in this experiment. Two of them were congenitally blind, and two were adventitiously blind. Their ages were 23 and 26 years and 21 and 34 years, respectively. They knew the shapes of the letters of the alphabet, as verified by a preliminary test in which they were asked to identify, using their fingers, the block capital letters of the alphabet in raised dots. They were asked to confirm the shapes of the alphabet further by touch. Then they had practice in order to familiarize themselves with this equipment. In the practice sessions, all the letters of the alphabet were arbitrarily divided into three subsets. ${ }^{2}$ The subjects were trained until they had acquired an identification accuracy of over $80 \%$ in each subset. 


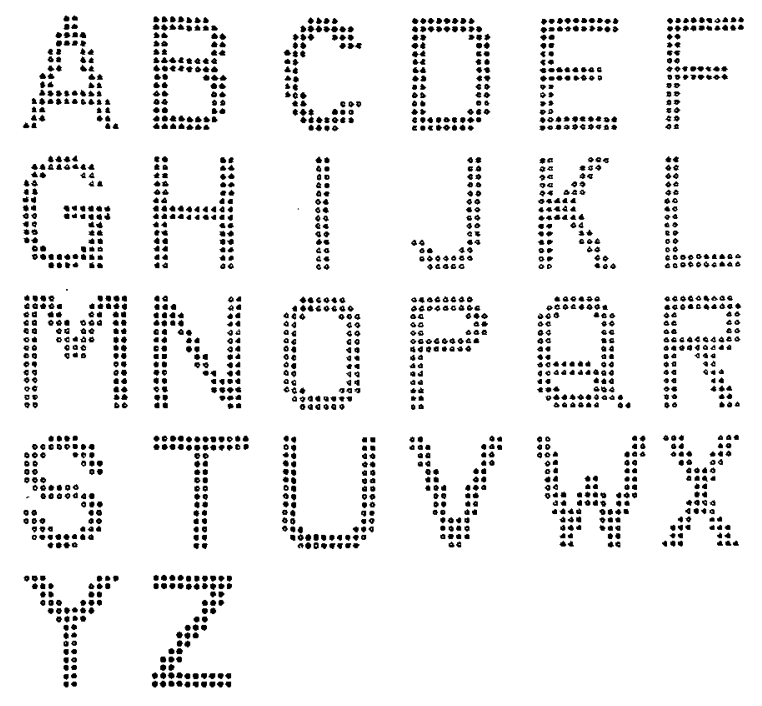

Figure 1. Schematic representation of the tactile letters used in the experiment.

\section{Procedure}

The subject, wearing a surgical gown, was asked 10 sit in the wheelchair, and was instructed not to lean back on the tactile matrix until the experiment started. After the experimenter had placed a visual display in proper position below the stationary camera, a verbal ready signal was given to the subject. Then the subject pressed back against the tactile matrix to feel the stimulation of the tactile pattern, and was given as much time as he needed to report which letter was displayed. If the report was incorrect, the experimenter informed the subject of the right one. Each letter of the alphabet was presented at random in each block of 26 trials. The number of trials per subject was not the same. The two congenitally blind subjects were run in 43 and 66 trials for each letter, respectively; the two adventitiously blind subjects had only 10 and 8 trials, respectively, as their personal schedules allowed.

\section{RESULTS}

In the experiment, 26 letters of the alphabet were presented at random in each block and error correction was given when the subjects misidentified. Thus, there was the possibility of learning during the experiment. Figure 2 presents the identification accuracies of the first 20 blocks of the experiment. The open and filled points in the figure indicate the results of the two congenitally blind subjects. The performances tend to increase up to about $90 \%$, although there are some fluctuations in performance. The two adventitiously blind subjects did not show any notable improvement in performance. Their identification accuracies remained at about $40 \%$, probably because they could not participate in the experiment regularly. The identification performance of even the congenitally blind subjects deteriorated after they had been away from the experimental situation for a length of time (more than 1 month). In such cases, performance deteriorated almost to the initial level, and tíen recovered again.
The general tendency of confusion seemed, upon inspection, to be about the same for each subject. ${ }^{3}$ Thus, the responses of the four subjects for each letter were pooled. The total number for each letter was 127 , on which the following analyses are based.

The results of the experiment are summarized by a confusion matrix shown in Table 1. The letters of the vertical column indicate stimuli, and those of the horizontal row, responses. Each cell value of the matrix, $f_{i j}$, represents the number of times that the response $\mathrm{j}$ occurred upon the presentation of stimulus $i$. The recognition accuracies for each letter are indicated in the last column. As can be seen, a wide range of recognition accuracies of letters was obtained. The best score was $100 \%$, with I as the stimulus, and the worst, $37 \%$, with $\mathrm{G}$. The average recognition accuracy for all letters was around $70 \%$.

Letters having the highest recognition accuracies were: I $(100 \%)$, J $(96.1 \%)$, L $(95.3 \%)$, A $(92.9 \%)$, and $\mathrm{T}(92.9 \%)$, which mainly consist of a few straight lines and are rather simple. The letters having the lowest recognition accuracies were: G $(37.0 \%)$, R $(42.5 \%)$, B $(44.9 \%)$, and S $(44.9 \%)$, which have curved or enclosing lines.

Some prominent asymmetries may be found in the confusion matrix. Subjects mistook $X$ for $Y$, whereas they never confused $Y$ with $X\left(f_{X Y}=14, f_{Y X}=0\right)$. The letters $N$ and $W$, and $E$ and $K$ also exhibited asymmetries $\left(\mathrm{f}_{\mathrm{NW}}=13, \mathrm{f}_{\mathrm{WN}}=3 ; \mathrm{f}_{\mathrm{KE}}=13\right.$, $\mathrm{f}_{\mathrm{EK}}=3$ ). However, there seems to exist a general symmetrical tendency such that strong confusions are apt to be symmetrical. Examples are: the confusion of stimulus $\mathrm{U}$ with response $\mathrm{W}$ and $\mathrm{W}$ with $\mathrm{U}$ $\left(f_{U W}=17, f_{W U}=17\right)$, the confusion of $D$ with $O$

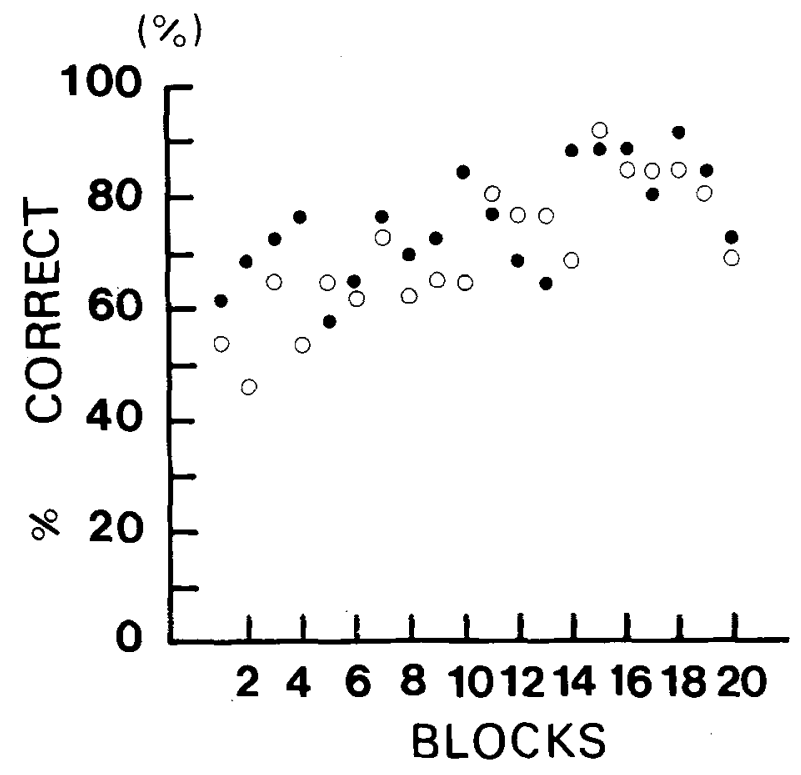

Figure 2. Percent correct responses of the first 20 blocks for two congenitally blind subjects. 
Table 1

Tactile Letter Confusion Matrix

Response

\begin{tabular}{|c|c|c|c|c|c|c|c|c|c|c|c|c|c|c|c|c|c|c|c|c|c|c|c|c|c|c|c|}
\hline & A & B & $\mathrm{C}$ & D & $E$ & $\mathrm{~F}$ & $\mathrm{G}$ & $\mathrm{H}$ & I & $\mathrm{J}$ & $\mathbf{K}$ & L & $\mathbf{M}$ & $\mathrm{N}$ & $\mathrm{O}$ & $\mathrm{P}$ & $\mathrm{Q}$ & $\mathbf{R}$ & $S$ & $\mathrm{~T}$ & $\mathrm{U}$ & $\mathrm{V}$ & W & $X$ & $\mathrm{Y}$ & Z & $\mathrm{p}$ \\
\hline 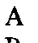 & 118 & 0 & 0 & 0 & 1 & 0 & 0 & 0 & 1 & 2 & 1 & 1 & 0 & 0 & 0 & 0 & 0 & 1 & 1 & 0 & 0 & 0 & 0 & 1 & 0 & 0 & .929 \\
\hline & 5 & 57 & 2 & 0 & 1 & 0 & 12 & 3 & 0 & & 6 & & 2 & 1 & & 0 & 11 & 10 & 3 & & & & 0 & & & & .449 \\
\hline & 2 & 2 & 71 & 1 & 11 & 1 & 5 & c & 0 & c & 9 & & 0 & 0 & 1 & 0 & & & 9 & & & & 1 & & 0 & & .559 \\
\hline U & 0 & 6 & 3 & 65 & 1 & 1 & 2 & 1 & 0 & c & 1 & 0 & 8 & 5 & 15 & 1 & 5 & 7 & 1 & & & & 1 & & 0 & & .512 \\
\hline & 2 & 2 & 11 & 4 & 78 & 7 & 7 & $c$ & 1 & c & 3 & 2 & 0 & 0 & & 0 & 0 & 3 & 3 & & & & 0 & & & & .614 \\
\hline & 0 & 1 & 0 & 0 & 4 & 100 & 1 & 0 & 1 & c & 1 & 0 & 0 & 0 & 0 & 10 & 1 & 0 & & & & & 1 & 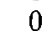 & & & .787 \\
\hline & 7 & 14 & 1 & 1 & 4 & 3 & 47 & 3 & 0 & c & 2 & c & 1 & 1 & 3 & & 18 & 11 & s & & ( & & 0 & & 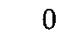 & & .370 \\
\hline & 1 & 3 & c & 6 & 0 & 0 & 1 & 68 & 0 & 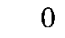 & 2 & & 3 & 12 & & & 5 & & 2 & & & & 0 & & . & & .535 \\
\hline & 0 & 0 & $c$ & c & 0 & & 0 & 0 & 127 & 0 & c & & 0 & & 0 & 0 & 0 & 0 & r & & 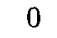 & ( & 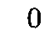 & 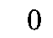 & 0 & ) & 1.000 \\
\hline & 3 & 0 & 0 & 0 & 0 & & c & c & 0 & 122 & 0 & & 0 & 0 & & 0 & 0 & 0 & ( & & 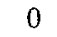 & & ) & & 0 & & .961 \\
\hline & 6 & 4 & 6 & 1 & 13 & & 2 & 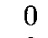 & 0 & & 65 & 1 & 0 & 1 & 0 & 4 & 2 & 4 & 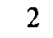 & & 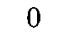 & & 0 & 5 & 3 & & .512 \\
\hline & 0 & 0 & 1 & 0 & 2 & c & 0 & 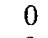 & 0 & $c$ & 0 & 121 & 0 & 0 & 0 & 1 & 0 & 0 & ( & 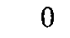 & & & 1 & 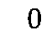 & 0 & & .953 \\
\hline & 0 & 1 & 0 & 5 & c & 1 & 1 & C & 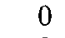 & 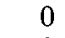 & c & 1 & 96 & 9 & 4 & 2 & 1 & c & & & 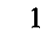 & 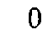 & $t$ & 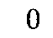 & & & .756 \\
\hline & 3 & 1 & 0 & 3 & 2 & 1 & 3 & 10 & 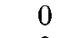 & 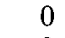 & 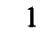 & & 4 & 63 & 4 & 1 & 9 & 1 & c & 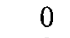 & & 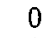 & 13 & 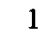 & & & .496 \\
\hline & 2 & 6 & 0 & 15 & 1 & 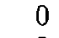 & 6 & 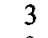 & & ( & & & 4 & 5 & 62 & 0 & 6 & 8 & 2 & & 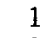 & & 0 & & 0 & & .488 \\
\hline & 1 & 0 & 0 & 1 & 0 & 3 & 2 & 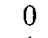 & & & 2 & & 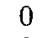 & 0 & 0 & 114 & 0 & 0 & 1 & & ( & & 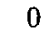 & & 0 & & .898 \\
\hline & 3 & 11 & 1 & 1 & 1 & 1 & 11 & & ( & & 3 & & & 3 & 7 & 0 & 59 & 1 & 3 & & & & 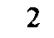 & & & & .465 \\
\hline & 3 & 9 & 1 & 2 & 2 & 4 & 11 & & , & 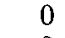 & 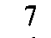 & & 1 & 3 & 4 & ( & 7 & 54 & 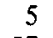 & & & & 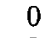 & 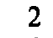 & 0 & & .425 \\
\hline & 3 & 9 & 3 & 3 & 1 & 0 & 15 & 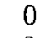 & 1 & & 4 & & & 3 & & & 6 & 11 & 57 & & & & & & & & .449 \\
\hline & 0 & 0 & 0 & 0 & 0 & 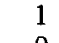 & 0 & c & 1 & & 0 & & . & 0 & & & 0 & 0 & 2 & 118 & 0 & 0 & & & & & .929 \\
\hline & 0 & 1 & 0 & 1 & 0 & 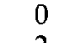 & 3 & 8 & & & 0 & & & & & & 0 & 2 & & 1 & 84 & 3 & 17 & & 0 & & .661 \\
\hline & 0 & 1 & 0 & 1 & 0 & 2 & 1 & 1 & & & 1 & & & 2 & & & 0 & 3 & & & 1 & 93 & & & 15 & & .732 \\
\hline & 1 & 2 & 0 & 3 & 0 & 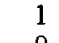 & 0 & 7 & & 0 & 1 & & & & & & 0 & 2 & & 0 & 17 & 4 & 85 & 0 & 0 & & .669 \\
\hline & 5 & 0 & 0 & 0 & 6 & ( & 1 & c & & & 6 & & & & & & 0 & 2 & & & & & 0 & 83 & 14 & & .654 \\
\hline & 0 & 0 & 0 & c & 0 & 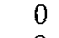 & 0 & c & & 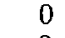 & 0 & & & & & & 0 & 1 & 0 & 6 & 0 & 8 & 0 & 0 & 111 & 0 & .874 \\
\hline Z & 0 & 0 & 1 & 2 & 1 & 0 & 0 & 0 & 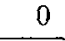 & 0 & 0 & & 0 & 0 & 0 & 0 & 0 & 1 & 2 & 1 & 0 & 0 & 0 & 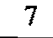 & 0 & 112 & .882 \\
\hline
\end{tabular}

Note-Letters in the first column are the stimuli. Total number of presentations of each letter is 127.

and of $O$ with $D\left(f_{D O}=15, f_{O D}=15\right)$, and the confusion of $B$ with $G$ and $G$ with $B\left(f_{B G}=12\right.$, $\left.\mathrm{f}_{\mathrm{GB}}=14\right)$. The correlation between all pairs of corresponding confusion values was .7 , which was highly significant at beyond the .01 level. These results may be attributed in part to the fact that the 26 letters of the alphabet were presented in a single block, and that errors were corrected after the presentation of each letter.

To analyze the confusion data, hierarchical clustering schemes (HCS; Johnson, 1976) and nonmetric multidimensional scaling (MDSCAL; Kruskal, 1964a, 1964b) were employed as Morgan (1973) advocated. Applications of both HCS and MDSCAL presuppose a symmetrical matrix of similarity or dissimilarity between the stimuli and responses. The conversion of the nonsymmetrical matrix of Table 1 into a symmetrical similarity matrix loses the directional information of confusion. However, the cells with higher confusion values generally have higher values in their counterparts, as indicated by the above correlation coefficient. Consequently, the conversion permits further analysis. A measure of similarity between letters $i$ and $\mathrm{j}, \mathrm{S}_{\mathrm{ij}}$, is defined by

$$
S_{i j}=f_{i j} / \sum_{i=1}^{n} f_{i j}+f_{j i} / \sum_{j=1}^{n} f_{j i},
$$

where $f_{i j}$ is the number of times that the response $\mathrm{j}$ occurred to the presentation of stimulus $\mathrm{i}$, and $\mathrm{n}$ is the number of stimuli.

The HCS method used to analyze the similarity matrix is a simple hierarchical clustering method, producing a tree, or dendrogram, each level of which shows a state of clustering. At the lowest level, each cluster contains just one object; at higher levels, the clusters at a lower level converge to make larger ones. At the highest level, all objects are contained in one cluster. This method may reveal some tactile confusion characteristics of this particular representation of the 26 capital letters of the English alphabet.

The results are shown in Figure 3, where the letters at the bottom of the dendrogram indicate the stimuli studied in this experiment. The numbers on the left side are the similarity values at which clusters appear. By considering horizontal sections of the dendrogram at different levels, different sets of clusters are obtained. As seen in Figure 3, the letter pairs U-W, $D-O$, and $G-Q$ became clusters at earlier stages than did others. This means that the letters in these clusters are strongly related and consequently easily confused with one another. The section of the dendrogram at the level of .15, for example, resulted in 17 clusters, B-G-Q-R-S, D-O, C-E, H-N, U-W, V-Y, and 11 others of just one letter each. This section shows an interesting set of clusters which is discussed later. 


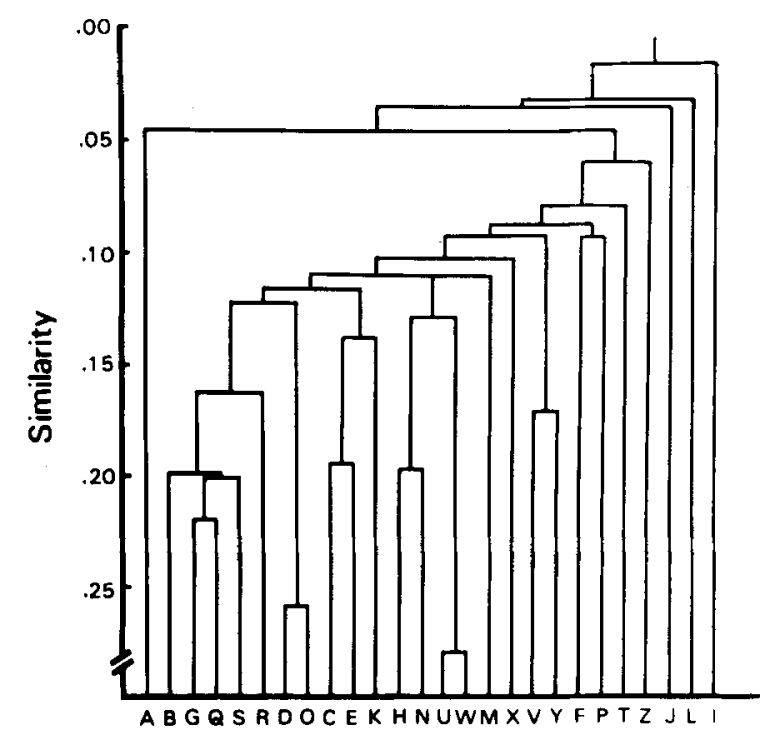

Figure 3. Hierarchical cluster analysis of Table 1.

Multidimensional scaling (MDSCAL) is a very useful method of organizing many stimuli along different dimensions. It has been successfully applied to find psychological characteristics of colors and visual forms (e.g., Indow \& Uchizono, 1960; Kikuchi, 1971). The MDSCAL analysis was employed in order to obtain some information about the possible dimensions of tactile letter confusion by the skin of the back. The MDSCAL analysis in the present study was applied by using a Euclidean metric and the primary approach to ties. That is, the interpoint distance between any two points, which represents similarity, is equal to the squared differences of their projections on each axis of the psychological space summed over all axes. In the primary approach, if two pairs of stimuli are ranked the same, a single ranking is allowed to have more than one interpoint distance in the space, whereas the more restrictive secondary approach requires that the interpoint distances for these pairs should be made equal (see Kruskal, 1964a, 1964b).

Figure 4 shows the index of fit or stress as a function of the number of dimensions. In the curve obtained, there is no abrupt change, such as an "elbow," that would help to determine the proper number of dimensions.

Though one to seven dimensional analyses were made, the three-dimensional solution was selected as an appropriate one on the basis of interpretability of the results and the shape of the stress curve. The three-dimensional solution indicated, however, rather a high stress value, i.e., $18.2 \%$, which is considered "poor to fair" according to Kruskal's verbal evaluation. But this evaluation is merely an empirical yardstick. The three-dimensional solution in two two- dimensional displays is shown in Figure 5. Figure 5a is the projection of the three-dimensional solution on the plane of the dimensions $\mathrm{I}$ and 11 , and Figure $5 \mathrm{~b}$ is the projection on the plane of the dimensions I and III. The results of the HCS analysis are also depicted in Figure 5. The encircled clusters are given at the level of about .15 (see Figure 3).

An examination of the configuration of the stimuli suggests that the first dimension may be identified as that of nonenclosing shapes vs. enclosing shapes, because letters such as I, T, and $L$ are at one end of the axis, while such stimuli as $Q, O$, and $B$ are at the other. On the second dimension, the letters $U, H$, and $W$ are associated with the highest values, whereas $\mathrm{C}$ and $\mathrm{E}$ have the lowest, so that this dimension may be considered that of vertical parallel lines or vertical lines. It might also be possible to consider this dimension cup-shaped at the top of the figure because the high-loading letters have a concave line at the upper part of the letters. At present, however, we tentatatively regard this dimension as vertical parallel lines. The letters which contain an accute angle in their shapes are given high values in the third dimension, and the letters $P$ and $F$, which contain a right angle, are at the other end of this dimension. $M$ is also high on this dimension, but this letter frequently

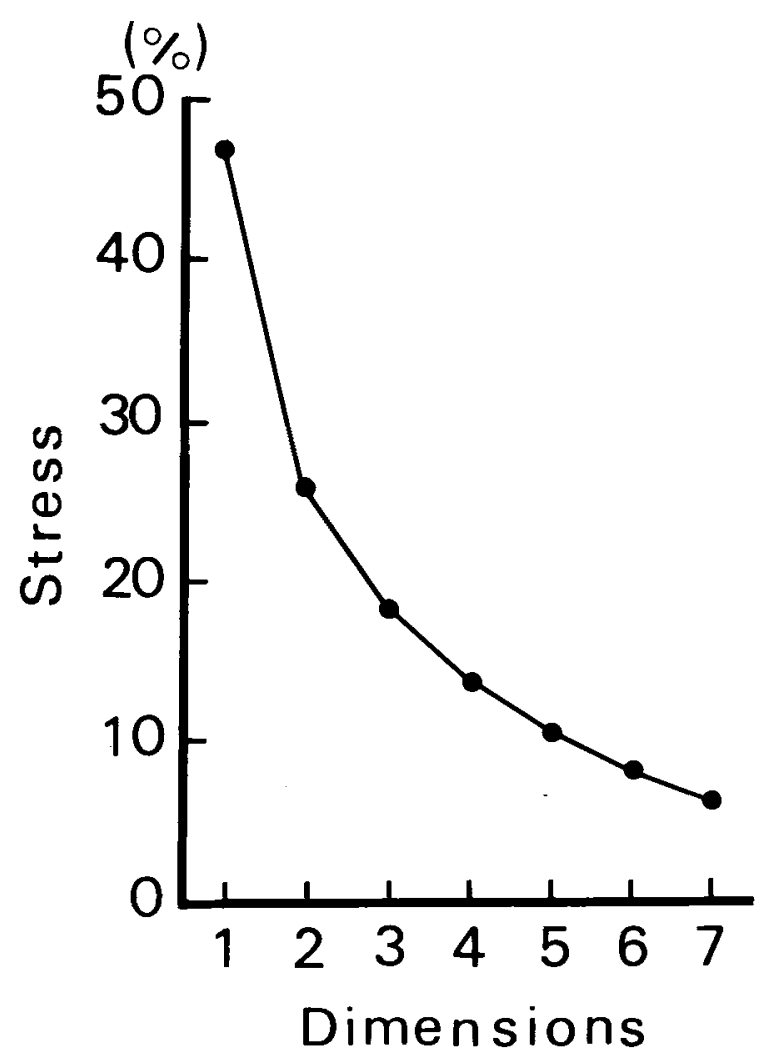

Figure 4. Stress as a function of number of dimensions. 

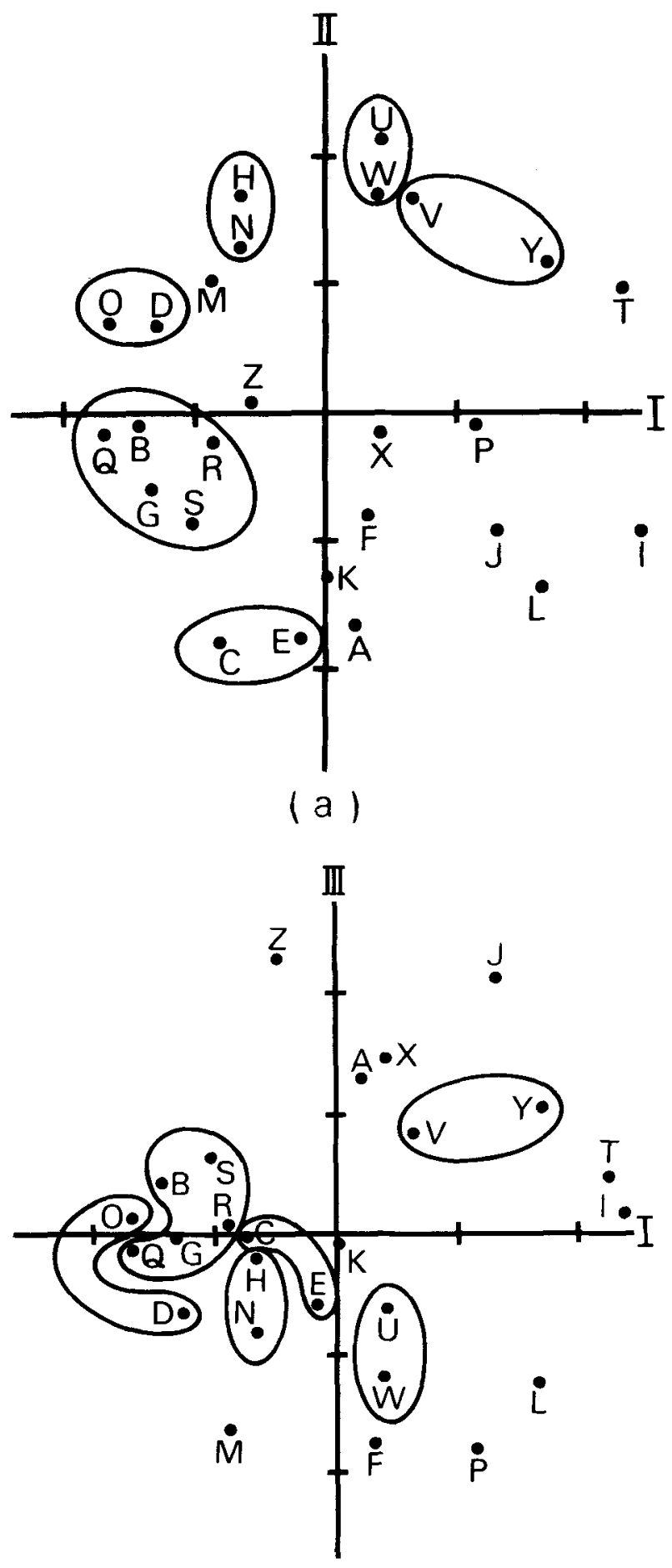

(b)

Figure 5. Three-dimensional MDSCAL analysis of Table 1. The encircled clusters are given at the level of about .15 (see Figure 3). (a) Projection on the place of Dimensions I and II. (b) Projection on the plane of Dimensions I and 111.

seemed to be perceived as an inverted $\mathrm{U}$, as discussed later. $\mathrm{J}$, which some may consider to contain a right angle, is on the other end of this dimension. This is probably because the subjects perceived something at the lower left-hand side of the vertical line, which immediately led them to the response of $\mathrm{J}$ because of its unique letter shape. It should be mentioned that some of the letters, at least, may not agree with the labels of the dimensions. It is obviously difficult to interpret the dimensions so as to fit all the letters.

It could be concluded, however, that at least three features, such as enclosing shapes, vertical parallel lines, and angle of lines, play an important part in recognizing tactile block letters of the alphabet by the skin of the back.

\section{DISCUSSION}

Craig (1974) investigated tactile letter recognition, using a 10 by 10 array of vibrators mounted on the back of a chair. Block letters of the alphabet were converted, by means of a 10 by 10 array of photocells, to tactile patterns. A thin handle was attached to each letter, so that the subject could move a letter across the array of photocells. Craig reported a confusion matrix, but only a limited set of the letters of the alphabet was used.

Loomis (1974) examined the effects of different modes of stimulus presentation, using the 400-point TVSS. A whole set of block capital letters of the alphabet was used in his study, as in the present study. One of his five modes of stimulus presentation, i.e., the full-field, stationary-letter mode, corresponded to ours. In this mode, a letter was presented all at one time, repeatedly and rapidly, on the surface of the skin of the back. Loomis reported a confusion matrix based on 42 judgments for each letter. The matrix showed that letters having the highest recognition accuracies were $\mathrm{I}, \mathrm{J}, \mathrm{L}$, and $\mathrm{T}$, and those having the poorest recognition accuracies were Q, G, D, N, R, X, and some others. The fullfield, stationary-letter mode yielded rather a poor average recognition accuracy, i.e., $34 \%$. This is very low in comparison with ours $(\mathbf{7 0 \%})$. This discrepancy may be due to the differences in the device and the procedures used. Loomis presented tactile letters to experienced and inexperienced subjects alike for only a short period of time $(1.5 \mathrm{sec})$, using the 20 by 20 point TVSS, whereas we presented them to experienced blind subjects for as much time as needed, using the 17 by 17 point TVSS. Moreover, in our experiment, movement of the subject's body was sometimes observed during the presentation of a tactile pattern. The introduction of body movement causes a change in the stimulated area of the surface of the skin, and prevents prolonged stimulation. Active body movement may allow the subject to sweep the tactile pattern, even if in a restricted manner, and he could extract more information from the tactile pattern presented. Despite these differences between Loomis' study and the present one, the cor- 
relation coefficient between Loomis' recognition accuracies and ours for all 26 letters of the alphabet was .72 , which is highly significant, beyond the .01 level. Thus, the results obtained by Loomis are quite similar to ours in relative accuracies among the letters. This fact suggests that some common mechanisms of tactile letter recognition exist in both studies, even though different styles of block letters and different procedures were used.

The encircled groups of letters in Figure 5 indicate letters in clusters having similarity measures greater than .15 in the HCS analysis. The proximity of the letters in each group in Figure 5 shows the consistency between the results of the two analyses, HCS and MDSCAL. The letters encircled are linked at a lower level of the dendrogram and become clusters at early stages. These clusters may throw a light on some underlying characteristics of tactile letter confusion.

The $\mathrm{H}-\mathrm{N}$ cluster and the U-W cluster suggest interferences in recognizing details in the inner parts between parallel lines. A difficulty in recognizing a short line within confining lines, such as the middle line of $\mathrm{E}$ in the C-E cluster, and another difficulty in discriminating between straight and curved lines, as illustrated in the D-O cluster, are also shown in some of these clusters. Similar observations were made by Craig (1974) concerning tactile letter recognition with the Kinotact. In a supplementary experiment, our subjects were asked to draw a tactile impression on a sheet of special paper used by blind people, which produces a raised pattern when written on with a pencil, in order to examine directly what kind of impression they had acquired. They frequently drew a pattern similar to $\mathrm{U}$ for $\mathrm{W}$ and a pattern similar to $\mathrm{C}$ for $\mathrm{E}$, as shown in Figure 6. The apparent loci of the lower parts of $\mathrm{W}$ were displaced and
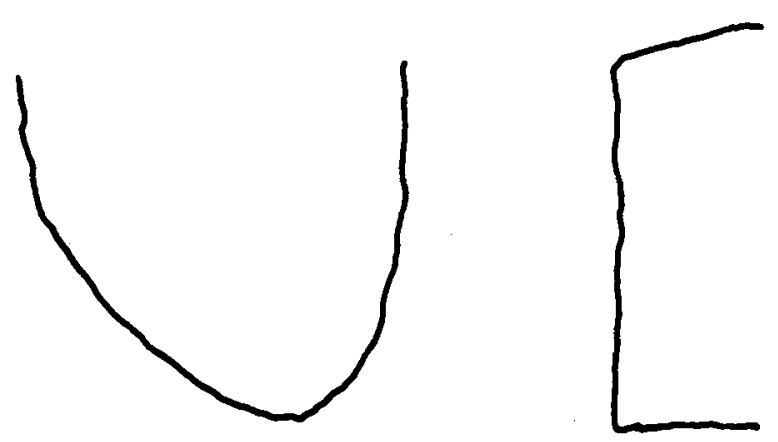

\section{W}

Figure 6. Examples drawn by the blind subjects when $W$ and $E$ were presented. represented by a horizontal line, and the middle horizontal line of $\mathrm{E}$ was missing. When $\mathrm{M}$ was presented, the inverted $U$ was drawn as in the case of $W$. These results are quite consistent with the confusion data obtained in the present study $\left(f_{W U}=17\right.$; $f_{E C}=11$ ). It is important to note that our subjects showed the other directional confusion also ( $f_{U W}$ $=17 ; \mathrm{f}_{\mathrm{CE}}=11$ ). This tendency could be attributed to the procedure used. Our subjects, as already stated, were informed of the correct letter when they misidentified, so that learning occurred as the experiment progressed. Moreover, each letter of the alphabet was presented only once in each block of 26 trials. Thus, they might have remembered the letters which had been presented at earlier trials. This may be the reason, in part, why such a high average recognition accuracy was obtained and, in part, why the error distribution was so limited.

It also seems difficult to discriminate two lines forming an acute angle, as in the letter $V$. The two lines of $\mathrm{V}$ can quite possibly converge into one line near the vertex and then $V$ will be immediately confusable with $Y$, as in the V-Y cluster. Because of their complicated structure, all members of the B-G-Q-R-S cluster were highly confusable with each other, so their recognition accuracies are very poor. It seems to be difficult for the subjects to detect gaps or line details in the transformed letter.

Craig (1974) and Loomis (1974) reported confusion matrices between block capital letters of the alphabet, but did not analyze their data further. Both HCS and MDSCAL analyses were applied to the Loomis data in the same way as in our study, because his procedure is somewhat similar to ours. The letter pairs V-Y, F-P, N-W, K-R, H-Q, and D-U became clusters at earlier stages in the HCS analysis. The analysis of his data also indicates the fusion of two lines, a difficulty in recognizing the inner details of confining lines, and difficulty in detecting gaps. Features, such as enclosing shapes, vertical parallel lines, and horizontal parallel lines, were extracted in the MDSCAL analysis. The results of the analyses of Loomis' data were similar to ours despite many differences in equipment, procedure, and style of letters. However, the consistency in his data was not as good when the results of the MDSCAL analysis were compared with those of the HCS.

To summarize, the results of the HCS analysis indicated that most of the confusion is evidently due to the displacement of the apparent loci, omission or fusion of loci, and failure to detect gaps or details in the letter shapes. The mutual influence of sensations produced by the tactile stimulators of letters impressed on the subject's back makes recognition of the finer details of a letter difficult. It can be concluded that letters are judged in terms of their basic 
features. The results of the MDSCAL analysis indicated that blind subjects utilize at least three independent basic features, such as enclosing shapes, vertical parallel lines, and angle of lines, when making their decisions.

\section{REFERENCES}

BACH-Y-RitA, P. Brain mechanisms in sensory substitution. New York: Academic Press, 1972.

Collins, C. C. Tactile television-Mechanical and electrical image projection. IEEE Transactions on Man-Machine System, 1970, MMS-11, 65-71.

Craig, J. C. Pictorial and abstract cutaneous displays. In F. A. Geldard (Ed.), Cutaneous communication systems and devices. Austin, Tex: Psychonomic Society, 1974.

Geldard, F. A. Cutaneous coding of optical signals: The optohapt. Perception \& Psychophysics, 1966, 1, 377-381.

In Dow, T., \& UChIzono, T. Multidimensional mapping of Munsell colors varying in hue and chroma. Journal of Experimental Psychology, 1960, 59, 321-329.

Johnson, S. C. Hierarchical clustering schemes. Psychometrika, $1967,32,241-254$.

KIKUCHI, T. A comparison of similarity space and semantic space of random shapes. Japanese Psychological Research, 1971, 13, 183-191.

KrUSKAL, J. B. Multidimensional scaling by optimizing goodness of fit to a nonmetric hypothesis. Psychometrika, 1964, 29, 1-27. (a)

Kruskal, J. B. Nonmetric multidimensional scaling: A numerical method. Psychometrika, 1964, 29, 115-129. (b)

Linvill, J. G., \& Bliss, J. C. A direct translation reading aid for the blind. Proceedings of the IEEE, 1966, 54, 40-51.

Loomis, J. M. Tactile letter recognition under different modes of stimulus presentation. Perception \& Psychophysics. 1974, 16, 401-408.
Morgan, B. J. T. Cluster analyses of two acoustic confusion matrices. Perception \& Psychophysics, 1973, 13, 13.24.

\section{NOTES}

1. Loomis (1974) studied tactile letter recognition under five modes of stimulus presentation, three of them employing a linear slit. In the slit modes of presentation, either the slit moved in front of the stationary letter, portraying the letter over the skin of the back (the moving-slit stationary-letter mode of Experiment I and the diagonal slit version of the moving slit stationaryletter mode of Experiment II), or the letter moved behind the stationary slit, entering successive slices of the letter on the same part of the skin (the stationary-slit moving-letter mode of Experiment I).

2. Our subjects were able to recognize the letters of the alphabet with their fingers, but they had difficulty in identifying them with their backs at first. Thus, we divided the letters into three subsets so as to make the alternatives of choice smaller. The subdivisions were: (1) D I J L O T U V X, (2) A F H K N P S Y Z, and (3) B C E G M Q R W. Although there was not any strict rule of division, Subset 1 consisted mainly of the letters of two strokes, Subset 2 of letters of three strokes, and Subset 3 of the remaining letters.

3. Generally speaking, the two adventitiously blind subjects showed a confusion tendency similar to that of the two congenitally blind subjects. Of course, there were confusion errors that only the adventitiously blind subjects made, and there were confusion errors that only the congenitally blind subjects made. Worthy of note is the fact that most of the confusion errors of the responses $\mathrm{I}$ and $\mathrm{J}$ were made by the adventitiously blind subjects. The congenitally blind subjects seldom confused $\mathrm{I}$ and $\mathrm{J}$.

(Received for publication June 26, 1978 ; revision accepted June $29,1979$. ) 